\title{
Question de genre et de stratégie discursive : quand la polémique rend le « débat » impossible
}

Jean-Michel Adam

\section{(2) OpenEdition}

Journals

\section{Édition électronique}

URL : http://journals.openedition.org/pratiques/3853

DOI : 10.4000 /pratiques.3853

ISSN : 2425-2042

\section{Éditeur}

Centre de recherche sur les médiations (CREM)

\section{Édition imprimée}

Date de publication : 1 juin 2013

Pagination : 233-248

\section{Référence électronique}

Jean-Michel Adam, «Question de genre et de stratégie discursive : quand la polémique rend le "débat » impossible », Pratiques [En ligne], 157-158 | 2013, mis en ligne le 18 décembre 2017, consulté le 01 mai 2019. URL : http://journals.openedition.org/pratiques/3853; DOI : 10.4000/pratiques.3853 


\title{
Question de genre et de stratégie discursive : quand la polémique rend le « débat » impossible
}

\author{
Jean-Michel Adam
}

Université de Lausanne \& Université de la Suisse italienne

\begin{abstract}
«Un esprit faux est un esprit très-borné : c'est un esprit qui n'a pas contracté l'habitude d'embrasser un grand nombre d'idées. Vous voyez par-là qu'il doit souvent en laisser échapper les rapports. Il ne lui sera donc pas possible de s'assurer de la vérité de tous ses jugemens. » Condillac (2002:8)
\end{abstract}

L'article de Driss Ablali (ci-après DA) se donne toutes les apparences d'un article instaurant un débat scientifique à propos des concepts de types, de genres et de généricité. Toutefois, la première moitié de cet article ne parle pas de ces trois concepts, mais s'emploie à mettre en cause les fondements épistémologiques de mes travaux selon des modalités discursives qui ne relèvent pas de la discussion scientifique.

\section{Quel est le genre de l'article de D. Ablali ?}

Dans le cadre d'un numéro consacré aux genres de discours, il est intéressant de questionner la nature générique de ce texte et le positionnement discursif de son auteur. Publié dans une revue comme Pratiques, ce texte devient éditorialement un article scientifique. Son auteur adopte d'ailleurs la posture adéquate : "Nous souhaitons, dans ce qui suit, contribuer à la réflexion portant sur les fondements épistémologiques et théoriques des notions de "type", de "genre" et "généricité”, telles qu'elles ont été théorisées en linguistique textuelle par J.-M. Adam ». Il déclare "croi[re] au débat académique » et il reconnaît que les travaux de celui qu'il vise "font autorité » en matière de linguistique textuelle. Il prétend même " avoir ressenti un réel plaisir intellectuel à suivre sur plus de trente ans l'évolution de ses positions théoriques et épistémologiques ». Mais suffit-il d'adopter cette posture et de prendre ces précautions oratoires pour entrer dans une véritable discussion scientifique? Suffit-il d'écrire "Si nous nous intéressons aux travaux d'Adam, ce n'est pas avec une visée polémique contrairement à certaines habitudes $d u$ débat académique », pour engager un véritable « débat » académique ? 


\subsection{Ceci n'est pas un « débat »}

Le titre de 1'article: «Types, genres, généricité en débat avec J.-M. Adam » opte pour le genre discursif du débat. C'est-à-dire pour un genre relevant de ce que Patrick Charaudeau (2013) appelle «l'échange interlocutif polarisé » ${ }^{(1)}$, qu'il distingue de "l'échange libre » et de «l'échange sollicité ». Ce type d'échange «établit un rapport antagoniste entre les interlocuteurs. Ils sont à la fois opposés l'un à l'autre et prennent des positionnements en contre de la position de l'autre et en pour de leur propre point de vue. La thématique est généralement unique et les participants à l'échange peuvent utiliser leur statut comme justifiant une autorité de savoir» (je souligne). Charaudeau identifie quatre genres «polarisés »: les genres de la dispute, de la discussion, du débat et de la controverse.

Comme il le montre, le genre débat est « difficile à déterminer, car l'usage qui est fait de ce terme brouille sa possible définition, toute confrontation de point de vue pouvant être nommée débat » (Charaudeau $2013: 34$ ). C'est exactement ce que fait DA. Au sens médiatique, on sait que le genre implique un cadre triangulaire engageant deux interlocuteurs ou plus, sous la direction d'un (ou plusieurs) animateur (s) et en présence immédiate ou distante d'un public. Le nom de genre choisi par DA introduit une certaine confusion, car lui et son interlocuteur n'ont pas été invités par Pratiques à débattre des questions de «types, genres et généricité ».

En effet, l'article de DA a d'abord été une conférence donnée à l'occasion d'une journée de l'association Conscila, à Paris, le 21 octobre 2011, à l'ENS, sur le thème "Modèles et théories du genre en confrontation ». Bien que le titre «Tous les textes ne sont pas des contes. Contre- "Propositions pour l'étude de la généricité" " et le résumé de l'intervention renvoyaient explicitement aux pages 11 à 20 du Texte littéraire. Pour une approche interdisciplinaire (Adam \& Heidmann 2009), aucun des deux auteurs n'avait été convié à « débattre » ${ }^{(2)}$. André Petitjean ayant pris la décision de publier les conférences de cette journée dans Pratiques, il a été convenu qu'en raison de l'indignation suscitée par l'intervention de DA, un droit de réponse était nécessaire et qu'il lui serait demandé de communiquer, pendant l'été 2012, un texte auquel pourraient être apportées les réponses nécessaires.

DA en est ainsi venu à parler de « débat » en modifiant, dans l'article, le titre initial de sa conférence. En terminant son «préambule » par une formule comme "nous dirons que l'essentiel de notre débat avec Adam portera sur [...] », DA s'institue lui-même en débattant et prend la communauté scientifique à témoin d'une confrontation plus proche de la dispute que de la discussion académique. Charaudeau donne cette définition du genre de la dispute:

Le genre Dispute correspond à un contrat d'affrontement (d'entrechoc, faudrait-il dire) de points de vue irréductibles. Le questionnement lui-même est sans base commune de partage, et les points de vue systématiquement opposés, les position-

(1) Je cite Patrick Charaudeau dans la pagination des épreuves de cet article à paraître. Les définitions sur lesquelles je m'appuie concernent les genres d'échanges interlocutifs oraux. Le déplacement en direction de l'écrit est donc de ma responsabilité.

(2) En fait de «contre-proposition », la conférence a rapidement révélé que DA s'en prenait plus à l'ensemble de mes travaux qu'aux propositions qu'Ute Heidmann et moi avions formulées. Comme la version définitive de son article prétend engager le « débat avec J.-M. Adam », je signe seul cet article. Les positions comparatistes et littéraires d'Ute Heidmann ne sont pas en cause et, si elle considérait DA comme un interlocuteur digne de ce nom, elle aurait d'autres arguments à faire valoir. 
nements antagoniques. Il s'agit ici de défendre, voire d'imposer à tout prix son point de vue et d'avoir raison. Les prises de paroles sont autoratifiées, souvent superposées, comme dans une lutte pour occuper l'espace de l'échange. La Dispute n'aboutit a priori ni à un accord, ni à un consensus, ni à un changement d'opinion : elle est un lieu discursif d'indécidabilité (ou « dialogue de sourds »). (2013:34)

De toute évidence, DA cherche à engager une dispute : il a prononcé une conférence qui est devenue cet article pour occuper l'espace de l'échange, s'y donner une place qui sert ses stratégies de positionnement dans le champ des sciences du langage. En désignant le partenaire de l'affrontement qu'il met lui-même en scène, il cherche à profiter de son statut de chercheur reconnu. La violence ad-personam des attaques force ce dernier à adopter une posture défensive et à ratifier son agresseur comme interlocuteur légitime.

Charaudeau définit tout autrement le genre académique de la discussion:

Le genre Discussion correspond à un contrat de confrontation de points de vue différents. À la différence de la Dispute, le rapport entre les interlocuteurs n'est pas a priori antagonique : il est différent mais non antagonique. La discussion porte sur un thème (ou plusieurs, mais successivement) et le questionnement, contrairement à la Dispute, implique que les interlocuteurs aient en partage un terrain commun. Les positionnements sont évidemment différenciés, et si chacun des interlocuteurs défend son point de vue et cherche à convaincre l'autre de la validité de ce point de vue, il existe des possibilités d'accord, y compris des changements d'opinion. Autrement dit, on se trouve dans un lieu de décidabilité (consensus) qui coexiste avec l'indécidable (dissensus) dont l'enjeu est de vérité. L'exemple emblématique est la discussion scientifique. (2013:34)

On ne peut mieux dire ce que l'on est en droit d'attendre d'une discussion scientifique ${ }^{(3)}$. Les propos tenus par DA laissent-ils entendre la possibilité d'un accord ? Cherchent-ils à convaincre et à faire éventuellement changer d'opinion l'interlocuteur désigné ? La possibilité du partage d'un terrain commun minimal à partir duquel une discussion aurait pu s'engager est-elle perceptible ? Les propos de DA se présentent-ils comme simplement différents ou comme radicalement et résolument antagoniques.

\subsection{Une généricité qui flotte significativement}

En dépit de son titre, la généricité de l'article de DA flotte entre la dispute et la controverse. Ce qui met singulièrement en péril une de ses affirmations péremptoires : « un article de revue ne peut se revendiquer de statuts génériques différents ». Certes pour ce qui est de « se revendiquer» auctorialement, mais cela n'entraîne pas l'adéquation de fait entre le mot et la chose ! La controverse est souvent confondue avec le débat et Charaudeau en propose la définition suivante :

(3) J'ai eu, dans un article du numéro 34 de Semen (Adam 2013), une vraie discussion qui prolonge le dialogue ouvertement, sincèrement et sérieusement engagé par André Avias sur «La macro-proposition : unité compositionnelle ». Dans cet article, je réponds techniquement à la proposition d'assimilation des concepts de période et de macro-proposition formulée par ce chercheur du Centre d'études supérieures d'Østfold, à Halden, en Norvège. Si je suis entré en matière, c'est parce qu'il engage, lui, une vraie discussion scientifique sur un point théorique et méthodologique précis, avec même des exemples à discuter. Sa posture et le contenu de ses observations rendaient possible la recherche de points d'accord et 1'exploration des désaccords. 
Le genre Controverse est [comme le débat] un échange polarisé, une confrontation de points de vue sur une scène triangulaire. Mais, d'une part, cette confrontation est limitée à deux points de vue (même s'il y a plusieurs participants) qui s'échangent à propos d'une seule question, d'autre part, elle se développe au regard d'un tiers évaluateur qui joue le rôle de référence. Il ne s'agit pas du public ni de l'animateur mais de ce qui permet d'évaluer la teneur des positionnements sur un certain questionnement : référence de savoir pour la controverse scientifique, référence morale pour la controverse sociale. Le contrat est donc d'établissement d'une vérité (même si l'on n'y parvient pas), ce qui rapproche ce genre de la Discussion. Mais à la différence de celle-ci, la Controverse dépasse les interlocuteurs : ils ne se trouvent pas dans une relation interpersonnelle, et ils savent que leur parole circule dans l'espace public. De plus, s'il y a controverse, c'est qu'il existe, a priori, des points de vue et des positionnements irréductibles les uns aux autres, ce qui n'est pas nécessairement le cas de la discussion. (2013:34-35)

DA limite la confrontation à deux points de vue : celui qu'il se charge de présenter comme faux et qui occupe dans son article la plus grande place, et le sien qu'il n'avance qu'en contrepoint et ne développe pas. Il érige en tiers évaluateur un savoir scientifique supérieur dit épistémologique et théorique qu'il convoque significativement à maintes reprises au début de son texte : "contribuer à la réflexion sur les fondements épistémologique et théorique des notions de "types", de "genre" et de "généricité" ", "l'évolution de ses positions théoriques et épistémologiques », "repérer le lien entre trois catégories descriptives : "type", "genre” et "généricité”, et leur socle épistémologique », «bases épistémologiques », « noyau épistémologique et méthodologique». Par la répétition insistante de ces termes dans le «Préambule » et dans la première partie, DA s'efforce de convoquer magiquement une instance supérieure qui le transformerait en juge porteur de la Vérité de La Science. C'est ainsi qu'il prétend, sans sourciller, «tenter d'évaluer la pertinence et la cohérence des propos tenus sur la place et le rôle des typologies textuelles dans l'analyse des discours et des corpus ». La modalisation par « tenter de » n'atténue pas la posture de grand évaluateur épistémologue autoratifié.

Charaudeau a eu la bonne idée de considérer un terme souvent employé, dans l'usage courant, en alternance avec débat et controverse : celui de polémique. Établissant une différence utile entre « genres » et «stratégies discursives », Charaudeau situe ces dernières dans « la marge de manœuvre dont dispose le sujet parlant à l'intérieur du contrat de communication, ce qui lui permet de s'individuer. Le sujet des stratégies discursives est un sujet libre de ses choix langagiers, conscients ou non conscients » $(2013: 36)$. La polémique n'est pas un genre, mais une stratégie discursive qui, dans l'article de DA, s'immisce dans le genre de la controverse et le transforme en dispute, empêchant ainsi toute discussion scientifique. Charaudeau propose cette définition qu'on croirait faite pour la circonstance :

La Polémique se manifeste lorsque, dans une situation interlocutive, un sujet s'emploie à mettre en cause la légitimité de parole de l'interlocuteur, soit en dénonçant son manque de statut légitimant (ad hominem), soit en attaquant sa position personnelle (ad personam). Ou alors c'est l'argument adverse qu'il tente de délégitimer [...]. Parfois même, il peut aller jusqu'à détruire l'argument adverse en le ridiculisant par ironie ou dérision. Cela explique qu'une polémique soit peu argumentée, qu'elle ne cherche ni le consensus ni la vérité, parce qu'elle est moins centrée sur la problématisation de la question que sur le positionnement de l'autre. On voit que la polémique relève d'un jeu stratégique de destruction verbale de l'autre qui est alors transformé en adversaire. (2013:36) 


\subsection{Un jeu stratégique de destruction verbale que confirment plan de texte et génétique}

Le dérapage polémique des propos de DA est lisible dans la structure de son article et dans le détail de certaines de ses formulations. Le premier paragraphe annonce un plan : "Plusieurs questions se posent et méritent qu'on s'y attarde. La première concerne le(s) cadre(s) théorique(s) dans lequel [sic] ces trois notions sont définies. La deuxième porte sur la transversalité des propositions élaborées par Adam et Heidmann pour l'étude de la généricité. Pour finir, nous mettrons l'accent sur la question des observables pour montrer qu'une caractérisation multi-sémiotique des genres ne peut se faire que sur corpus ». Cette annonce du plan de texte d'un article en trois parties ne correspond que très faiblement aux trois parties de la structure manifeste : la première question recouvre les deux premières parties : "Des séquences textuelles aux genres : quelle(s) épistémologie(s) ? » et « Des types aux genres »; la deuxième question recouvre manifestement le point 3 : «Six propositions pour la "généricité" »; quant au « pour finir», il se réduit à moins d'une page pompeusement intitulée « Perspectives » et qui n'apportent rien de plus au « débat ». Ce flottement du plan de texte est révélateur d'une absence de ligne argumentative claire. Les 6 premières pages («Préambule(s)» compris) jouent un rôle si important que la partie sur les « Six propositions », plus propice à ce qui aurait pu être une discussion, ne peut donner lieu à un échange scientifique serein et constructif.

La première version écrite de l'article de DA portait déjà un titre différent de celui de la conférence, mais ne reflétait pas son contenu réel : "Types, genres, généricité. Éléments de lecture pour une typologie sur corpus ». Les responsables de la rédaction de Pratiques, Caroline Masseron et André Petitjean, ont exigé une révision de ce texte qu'ils ont jugé excessivement polémique et de surcroît brouillon dans son écriture et son argumentation. C'est ainsi que, dans la version qu'on vient de lire, des jugements comme «Les soubassements épistémologies d'Adam sont embrouillés » sont plus aimablement devenus, par une opération de remplacement de la fin de l'énoncé : «Les soubassements épistémologies d'Adam ne sont pas faciles à cerner ». Le reproche d' "impuissance heuristique à tirer toutes les conséquences de ce qui définit la généricité : impuissance liée à l'empan de données relativement étroit sur lequel reposent leurs travaux des dix dernières années, limités au texte littéraire et au genre du conte » a été charitablement supprimé, ainsi qu'une phrase comme «Au lieu de se contenter d'énumérer des universaux pour découvrir la pierre philosophale ». Une phrase du «Préambule»: «Si nous nous intéressons aux travaux d'Adam, ce n'est pas, contrairement à certaines habitudes du débat académique, pour monter sur nos grands chevaux, comme on pourrait le croire » est devenue après remplacement et déplacement significatifs : "Si nous nous intéressons aux travaux d'Adam, ce n'est pas avec une visée polémique contrairement à certaines habitudes du débat académique ».

Ces quelques corrections de surface et de rares modifications ne changent rien au fond des attaques d'un article avec lequel il est difficile d'engager la discussion. Outre le manque de rigueur dans la conduite de l'argumentation globale, les négligences de détail sont multiples.

DA parle systématiquement de «genre textuel» et de «typologies textuelles », sans expliciter ses choix terminologiques. Pour ma part, j'ai très tôt expliqué pourquoi je rejetais les «typologies textuelles». Je ne parle jamais de « genres tex- 
tuels », mais toujours de « genres de discours » parce que, comme Rastier, je pense que les pratiques discursives (discours religieux, publicitaire, politique, littéraire, syndical, etc.) donnent lieu à des genres et sous-genres discursifs. De façon peu élégante, DA me reproche (au point 3.3.) de ne pas adopter cette position, mais dans le temps même où il me fait la leçon, il utilise le terme «genres textuels » qui n'est pas en cohérence avec la position de Rastier qu'il suit pourtant par ailleurs. Le choix terminologique de DA est plus proche de celui de chercheurs comme Jean-Paul Bronckart ou Luiz Antônio Marcuschi, mais eux prennent soin d'expliquer pourquoi ils parlent de « genres textuels » et ils argumentent leur position. DA se dispense de cet effort.

Je reconnais, en revanche, qu'il a parfaitement raison de critiquer le titre d'un de mes articles anciens (1998), dans un volume à orientation littéraire destiné aux agrégatifs : «Les genres du discours épistolaire ». Le mot discours était là improprement employé. Comme je parle de genres de récit, de genres de l'argumentation ou de genres descriptifs pour désigner des catégories de mise en texte qui traversent les pratiques discursives («polyprax » dirait DA), je parle, pages 203-210 de la nouvelle édition de Les textes : types et prototypes, sans avoir attendu la remarque de DA, de "genres de l'épistolaire », effectivement différents dans les discours littéraire, journalistique (courrier des lecteurs et lettre ouverte), médical, familial, administratif, etc. Le juste reproche de DA n'est, une fois encore, malheureusement pas nuancé par ce que j'ai écrit par la suite.

Lorsque DA se lance, au début de la section 3.1, dans une démonstration à propos d'une référence à Derrida, il ne prend pas la peine de se demander pourquoi et comment nous citons, Ute Heidmann et moi, un philosophe de la déconstruction fort éloigné de nos positions épistémologiques. Si nous le citons, c'est précisément parce qu'il est surprenant que ce philosophe déconstructionniste formule dans des termes qui conviennent à notre démonstration une conception des genres que nous étayons par les études de la suite du livre. Considérer, par principe, que ce que dit un philosophe est linguistiquement impertinent me paraît être une position simpliste et une négation de tout le travail d'ouverture interdisciplinaire d'un ouvrage dont le titre et le propos est explicite : Pour une approche interdisciplinaire. Manifestement, DA mélange les arguments et, pour une fois qu'il fait un effort de retour aux textes cités (en l'occurrence il semble avoir relu l'article de Derrida), il ne prend pas sérieusement en considération le livre dans lequel les thèses sont présentées et le travail effectué sur les concepts.

DA m'attribue une définition de la généricité qui est, en fait, tirée d'un article écrit avec Ute Heidmann (au point 3, paragraphe 4, à propos de 2004 : 62). En corrigeant les états successifs de son article, il aurait pu vérifier ses références bibliographiques : Adam 2004 recouvre exactement Adam \& Heidmann 2004 et cette première référence est tout simplement fausse. De plus, il se laisse aller à écrire :

Tout cela dans un nouveau paradigme que les auteurs appellent maintenant « Analyse textuelle des discours », que l'on pourrait appeler " analyse textuelle du discours littéraire ", car toutes les propositions qu'Adam \& Heidmann [...] ont développées dans les dix dernières années, l'ont été essentiellement sur un seul discours, le discours littéraire.

Cette nouvelle affirmation est doublement erronée. D'abord la théorie de l'analyse textuelle des discours me revient exclusivement et Ute Heidmann, qui développe quant à elle une analyse comparative des discours littéraires, ne se reconnaî- 
trait aucunement dans la perspective linguistique qui est la mienne. Ensuite, cette théorie de l'analyse textuelle des discours est développée dans un livre qui n'est pas du tout centré sur les textes littéraires et la théorie exposée dans ce livre est appliquée à de nombreux textes publicitaires, journalistiques et politiques.

Le manque de rigueur de cet article pourrait nous laisser croire qu'il a été bâclé, mais il a pourtant été réécrit plusieurs fois par son auteur !

\section{Critique d'une stratégie discursive}

\subsection{La sous-information comme moyen de déformation stratégique}

La notion de " généricité », que j'ai largement réutilisée dans Genres de récits (Adam 2011a), doit énormément à Ute Heidmann. Nous avons, en effet, pensé ensemble la question des régimes de généricité auctorial, lectorial et éditorial (auxquels elle a proposé depuis d'ajouter un régime traductorial sur lequel elle a beaucoup travaillé). Les propositions théoriques que critique DA sont le résultat de dix années de travail sur un corpus constitué par les contes en vers et en prose de Perrault, les Kinder-und Hausmärchen des Grimm, les premiers contes d'Andersen, diverses réécritures parodiques ou continuations et traductions dans plusieurs langues. En conséquence, le reproche d'absence de travail sur corpus émis par DA est déjà injuste et irrecevable pour cette seule partie des travaux menés avec Ute Heidmann. Si DA avait fait l'effort de dépasser la page 23 de notre livre de 2009 et s'il avait vraiment lu Le texte littéraire en entier et Textualité et intertextualité des contes. Perrault, Apulée, La Fontaine, Lhéritier (2010), il aurait pu porter un jugement plus informé et objectif.

Cette falsification sélective des données est visible dès le tout début de 1'article, quand DA précise qu'il ne vise pas «l'ensemble des travaux d'Adam, tels qu'ils portent sur le style, l'argumentation ou l'analyse des discours de la presse ». De cette manière, il croit se dédouaner par une décision arbitraire qui détruit la cohérence de mes travaux : j'ai commencé ma réflexion sur les genres dans l'édition 1999 de La linguistique textuelle et je n'ai avancé, avec Ute Heidmann, nos propositions sur la généricité des textes qu'après avoir moi-même travaillé pendant des années sur l'argumentation publicitaire (Adam \& Bonhomme 1997 [2011] \& 2000) et politique (Adam 2004, Adam \& Herman 2000 \& 2004, Adam \& Pahud 2010), après avoir consacré une longue recherche aux genres de la presse écrite (codirection, avec Thierry Herman et Gilles Lugrin, des numéros 94 de Pratiques sur les « Genres de la presse écrite» (1997), 13 de Semen: «Genres de la presse écrite et analyse de discours » (2000), et 3-4 d'Études de Lettres 2000 : «La presse écrite : des genres aux mélanges de genres »; voir aussi Adam 1997b), après avoir enfin longuement étudié les genres $d u$ conseil et $d u$ dire de faire (Adam 2001a \& 2001b; repris dans 2011b : 225-264).

C'est en croisant les résultats obtenus à propos de discours non littéraires avec ce qui se dégageait des travaux menés avec Ute Heidmann sur le cas particulier des contes que j'ai défendu les positions visées dans la dernière partie de l'article de DA.

Il est trop facile d'invoquer le caractère littéraire du corpus de travail en passant sous silence les travaux que je viens de mentionner. Cette forme de mauvaise foi m'avait indigné en octobre 2011 et me révolte encore aujourd'hui, même à la lecture de la note ajoutée à la suite de mes critiques de la première version écrite de cet article. Je cite cette note 8 dont la perversité n'est peut-être pas évidente : 
Ici on pourra rappeler que ces six propositions sont placées dans l'introduction du texte Littéraire. Pour une approche interdisciplinaire, emplacement qui sert de fondation à l'analyse empirique de tous les discours littéraires convoqués dans cet opus. Adam a travaillé sur plusieurs types de discours, personne ne peut le contester : le discours politique, journalistique, publicitaire, procédural, etc. Mais sa réflexion depuis les années 2000 a fait le choix de se placer essentiellement dans le giron du discours littéraire. (Sic pour la ponctuation et les majuscules du titre de 1 '« opus » incriminé)

La seconde phrase balaie d'un revers de manche la réfutation que j'avais communiquée à DA à la lecture du premier état de son article : depuis le passage au $\mathrm{XXI}^{\mathrm{e}}$ siècle, je me serais placé dans «le giron du discours littéraire ». N'importe quel lecteur un peu honnête sera pourtant sensible au fait que j'ai, dans mes trois derniers livres, équilibré les exemples de textes littéraires et de textes non littéraires d'origines discursives diverses. C'est le cas des deux études qui concluent $L a$ linguistique textuelle 2011 : les appels du 17 et du 18 juin 1944 du Maréchal Pétain et du général de Gaulle sont suivis d'une étude d'un petit texte de Jorge Luis Borges et de la problématique de sa traduction; Genres de récits est composé de deux chapitres consacrés à de genres narratifs littéraires : le poème narratif et le monologue narratif au théâtre, et de deux chapitres consacrés l'un au fait divers et l'autre au récit politique. J'ai déjà parlé de l'ajout du chapitre 6 des Textes : types et prototypes 2011, consacré aux genres procéduraux qui est pour moi très important.

En consultant la bibliographie de mes travaux des années 2000 à 2012 portant sur des corpus non littéraires, les lecteurs de Pratiques pourront eux-mêmes juger l'impertinence de 1'affirmation selon laquelle depuis les années 2000, j'aurais fait le choix de me placer essentiellement dans le giron du discours littéraire. Même si je pense, avec Henri Meschonnic, que la traduction et le discours littéraire sont des postes d'observation du langage essentiels pour les linguistes, mes propositions sont issues d'un travail de fond sur des corpus largement « homoprax » et «polyprax », pour reprendre la terminologie de DA.

La note 3 de l'article de DA est également révélatrice d'une façon de (ne pas) travailler : "Ici il faut souligner que c'est dans la thèse de V. Portillo Serrano, Problématique des genres dans les productions écrites universitaires : cas du résumé scolaire chez des étudiants français et mexicains, soutenue à l'Université de Franche-Comté en 2010, que nous avons rencontré plusieurs citations tirées des travaux d'Adam qui nous ont permis de mieux appréhender les questions théoriques et épistémologiques traitées dans cette contribution ». En signalant honnêtement qu'il cite de seconde main, DA montre surtout qu'il n'a pas pris la peine de (re)lire ce que j'ai écrit. Il aurait pu, si c'était un vrai chercheur, trouver lui-même ces citations et d'autres encore, au lieu de se contenter de citer de seconde main une jeune chercheuse débutante.

Une partie de la critique porte sur mes rapports avec la psychologie cognitive et en particulier avec les travaux de Walter Kintsch et Teun A. van Dijk associés. Au sein d'un éphémère Groupe TETA, Michel Charolles, Jean-Paul Bronckart, François Rastier, moi et quelques autres, nous avons, à la fin des années 80-début 1990, beaucoup travaillé avec le laboratoire de Dijon que dirigeait Michel Fayol, avec Guy Denhière, avec le laboratoire de Poitiers d'Esperet, Gaonac'h, Passerault et Coirier ou encore celui d'Annie Piolat, à Aix-en-Provence. La référence aux recherches menées alors sur la lecture et sur les théories psycho-linguistiques du texte est disqualifiée par DA, au nom d'une incompatibilité de principe entre 
ces travaux et mes références à la grammaire de texte allemande. C'est pourtant à partir de là que s'est faite l'évolution de la linguistique textuelle comme discipline et, plus largement, de la linguistique transphrastique. Dans les années 1975-1990, nous étions quelques-uns, dans Pratiques, à considérer que la linguistique ne pouvait rester en dehors de la forte demande sociale de l'époque en matière d'échec en lecture et de production écrite. C'est ce qui a motivé, chez moi comme chez Michel Charolles, la volonté de développer la linguistique textuelle en engageant un dialogue avec les recherches expérimentales en matière de lecture, compréhension et production écrite.

Dans Dialogue, avec Krystyna Pomorska, Roman Jakobson insiste sur un fait qui justifiait un tel travail. Comme le disait Jakobson, beaucoup de questions linguistiques attendaient d'être réexaminées à la lumière des contraintes cognitives :

Tel est le procès qui réunit en ensembles toujours plus larges les éléments qui, échappés à la perception immédiate, appartiennent déjà à la mémoire immédiate les sons en mots, les mots en phrases et les phrases en énoncés entiers.

Le rôle de la mémoire à court terme et à long terme constitue l'un des problèmes centraux, dirais-je, de la linguistique générale et de la psychologie du langage dans ce domaine aussi, beaucoup de questions sont à revoir et à réexaminer avec plus de précision [...]. (1980:72)

DA ne fait pas l'effort de recontextualiser certaines prises de position. Lorsqu'il cite des articles publiés dans Pratiques en 1975 et en 1979, il les décontextualise du travail militant qui était alors le nôtre et il ironise sur le grand écart entre travaux d'inspiration matérialiste de l'époque et d'inspiration sémiotique. L'une des études qu'il cite (1975), ma première publication sur le discours publicitaire, visait alors à légitimer l'introduction de ce genre d'icono-textes en classe. Ce qui peut paraître banal aujourd'hui ne l'était pas du tout en 1975 ! Il s'agissait de montrer que ces textes pouvaient être des objets de lectures et d'apprentissages engageant des savoirs et des procédures de distanciation critique. L'autre article était un essai de prise en compte des tendances du nouveau roman à abandonner le récit au profit d'autres modes de construction, en particulier poétiques. La sémiotique était alors traversée de courants différents et certaines propositions de Julia Kristeva étaient stimulantes. Tout cela fait sens dans la perspective du combat que nous menions, à l'époque, pour changer la conception du canon littéraire et le littéraro-centrisme de l'enseignement du français. C'est ce dont témoigne également le livre que j'ai écrit avec Jean-Pierre Goldenstein, en 1976 : Linguistique et discours littéraire ; livre dont je serais tenté de renier aujourd'hui certains développements théoriques très datés et encore naïfs (comme celui sur la grammaire de texte ou l'analyse de la publicité Mir Rose), mais pas le chapitre sur les anagrammes de Saussure, par exemple.

\subsection{Quand l'ouverture aux sciences des textes devient « instabilité épistémologique "}

Faute de temps, je me concentre sur ce que DA nomme pompeusement une « reconstitution épistémologique » de mes travaux et la conclusion à laquelle il aboutit : «[...] un trait reste inchangé. Avant d'aller plus avant, un premier constat s'impose : chaque décennie remet en cause le fondement théorique et épistémologique de la décennie précédente. Ce que les années 2000 ne vont pas démentir, et de façon répétitive [...] ». Ces revirements compulsifs qui découperaient mes travaux en cycles de dix ans : 1970, 1980, 1990, 2000, 2010 sont mis au compte d'une 
" absence de cohérence au niveau des postures théoriques ». Jugement dont les lecteurs ont certainement mesuré 1'amabilité évaluative. À mon « incohérence », DA ajoute une instabilité épistémologique : «il est très difficile de trouver dans la réflexion d'Adam sur les typologies une stabilité épistémologique. Sa conception du texte et du discours $n$ 'a pas cessé d'évoluer mettant à l'épreuve toute tentative de cerner son socle épistémologique et méthodologique ». Il est vrai que, pour comprendre l'évolution de mes travaux, il faudrait les lire de près et prendre connaissance du contexte théorique des années considérées.

À titre de comparaison, je renvoie au travail de lecture entrepris récemment par Guy Achard-Bayle, qui montre la cohérence de mes travaux sur le texte et le discours de 1985 à 2011. Sa contribution met en cause la simpliste et paresseuse division en décennies de DA. Achard-Bayle, qui m'a vraiment lu, distingue le débat que je mène entre 1985 et 1992 sur les typologies de texte et la façon dont je les mets en cause dès la première édition de Les textes : types et prototypes (1992). Il montre comment les concepts clés de linguistique textuelle de « cohésion-cohérence " sont déjà présents dans Le Texte narratif (1985). Entre 1989 et 1999, Achard-Bayle rappelle mon hésitation entre " pragmatique (du discours) vs linguistique (du texte) » sans la caricaturer. Je m'en explique d'ailleurs dès la première version de La linguistique textuelle. Des genres de discours aux textes (1999) qui marque, par rapport à mes Éléments de linguistique textuelle (1990), un déplacement de la grammaire de texte vers une linguistique du texte et du discours. Achard-Bayle a raison de distinguer enfin une période de 2005 à 2011 qu'il intitule « Des textes aux discours » où j'affirme résolument l'inscription de la linguistique textuelle dans l'analyse de discours, en la mettant au service de l'analyse de la diversité des pratiques discursives. Si l'on en croit Achard-Bayle, cette évolution s'est faite sans incohérence épistémologique, mais au contraire avec une volonté de construire et de (re)définir les fondements théoriques et les concepts clé d'une linguistique textuelle à la française.

Je renvoie également au chapitre des Grandes théories de la linguistique que Marie-Anne Paveau et Georges Elia Sarfati (2003 : 184-194) consacrent à mes travaux et à leur évolution, et, plus récemment, à ce début d'un compte-rendu de lecture de Genres de récits par Jacques-Philippe Saint-Gerand, dans le numéro 22 de la revue Questions de communication (2012):

Depuis 1984, Jean-Michel Adam, qui a choisi Lausanne comme poste d'observation des débats franco-français et internationaux portant sur les aspects linguistiques de la littérarité, à travers plus d'une dizaine d'ouvrages et d'innombrables articles, a édifié sur cet objet une œuvre considérable qui se signale par son acuité et sa densité. Une œuvre qui, prenant en compte les travaux développés sur les mêmes objets par d'autres chercheurs, se signale aussi par sa cohérence intrinsèque et sa cohésion formelle, qui pourrait donc être définie comme une « entité autonome de dépendances internes » c'est-à-dire comme une structure selon la définition de Louis Hjelmslev. Cette dimension du travail de l'auteur est marquée ici par le retraitement ou le recyclage de textes antérieurement publiés, sur lesquels se pose un regard autocritique à des fins mélioratives.

Michèle Monte parle, elle aussi, d'évolution sur fond d'un projet qui reste constant :

Jean-Michel Adam a évolué dans sa façon de penser les relations entre texte et discours tout en restant fidèle à un projet : derrière les variations infinies des discours, saisir des éléments de stabilité, de régularité, dans la composition et le fonctionnement des textes. (2013, à paraître) 
Pour saisir la « fidélité à un projet » dont parle Monte ou la « cohérence intrinsèque et [1] a cohésion formelle » dont parle Saint-Gerand, il faudrait effectivement prendre en compte l'ensemble de mes travaux, ce que DA ne fait pas et ce qui lui permet, de diagnostiquer chez moi une forme pathologique d'instabilité épistémologique.

Cette instabilité épistémologique tiendrait au fait que j'ai travaillé sur des concepts narratologiques d'origine sémiotique et sur des concepts d'origine psycholinguistique dans mes travaux sur les textes narratifs, qui convoquent aussi bien Propp et Greimas, que Bremond et Labov. Elle tiendrait au fait que j'ai travaillé des concepts poétiques de Philippe Hamon à côté de concepts rhétoriques et linguistiques, dans mes écrits sur les textes descriptifs; de concepts sémiologiques (travaux de Grize et Borel) et rhétoriques (Perelman et Toulmin) dans mes travaux sur l'argumentation et l'explication. Que cette impression résulte d'une lecture superficielle et peu informée, passe encore, mais comment ne pas reconnaître que je retravaille les concepts d'origines théoriques diverses, dans le but de leur donner des fondements linguistiques, en les reformulant et en les redéfinissant ? L'emprunt à des travaux de pointe sur des objets proches devient ainsi transformation et intégration dans un cadre théorique qui s'enrichit mais reste obstinément celui d'une linguistique textuelle dont les contours s'affinent et les concepts opératoires également. Ceci au prix d'un travail de recherche et de remise en cause continues, et tant pis si cela dépasse DA, dont la conception fixiste de la science est assez inquiétante, naïve et, pour tout dire, navrante.

Lorsqu'il cite une phrase comme : « À ce niveau de la recherche, il nous semble nécessaire que la grammaire de texte intègre une part de la grammaire de récit. [...] Les recherches de A.J. Greimas, T. Todorov et surtout C. Bremond peuvent être reconsidérées ici » (Adam \& Goldenstein 1976), DA n'y voit qu'incohérences et soubassements épistémologiques embrouillés. En fait, le verbe « reconsidérer» et le cadre fixé de la "grammaire de texte » (état théorique de la discipline au milieu des années 1970) confirment le fait que c'était dans un cadre théorique donné que les concepts narratologiques et sémiotiques étaient alors reconsidérés par moi, déplacés et redéfinis. Mon attitude épistémologique a donc toujours consisté en un (re)travail systématique des concepts.

Si je dénonce les emprunts éclectiques et « outilitaires » de certains de mes collègues (en particulier de certains ouvrages de stylistique et d'analyse textuelle), c'est précisément parce que mon propos a toujours visé à constituer un cadre théorique au sein duquel puissent être repensés et retravaillés des concepts issus de disciplines des textes certes non linguistiques mais voisines et aussi fécondes que la poétique, la rhétorique, la stylistique, la narratologie, la sémiotique, la sémiologie de Grize, la théorie de l'argumentation, la génétique, la philologie, 1'interactionnisme social de Bronckart ou la sociolinguistique du Labov des années 1970. Bien sûr les épistémologies, méthodologies, corpus et histoires de ces disciplines et champs de recherche sont hétérogènes et étrangers les uns aux autres, mais c'est ce que j'interroge. Lorsque j'écris que «L'ancrage de la linguistique textuelle dans l'analyse de discours redistribue les rapports entre sciences du langage, sciences ou disciplines des textes littéraires, sciences de l'information et de la communication », un lecteur attentif comme Jean-Marie Viprey en tire un commentaire très différent des jugements de DA : "On reconnaît là la haute conscience que Jean-Michel Adam a cultivée et transmise de l'importance d'une attention soutenue au devenir des champs d'intervention des savants, à la socialité des disciplines et à l'ouverture épistémologique » (2011: 184). 
Mon ambition de poursuivre le projet de «translinguistique des textes, des œuvres » de Benveniste en fondant une linguistique textuelle de langue française constitue le fil rouge de mes travaux, même dans le domaine de la stylistique. Le dialogue avec des disciplines des textes étrangères à la linguistique est indispensable pour avancer. C'est à fédérer les sciences et disciplines du texte que je travaille depuis plus de trente ans et quand je participe à des recherches interdisciplinaires, avec des anthropologues, des philosophes, des littéraires, des spécialistes des médias, de philologie, de traduction ou de génétique des manuscrits, je le fais toujours, et c'est même précisément là la difficulté, à partir du cadre théorique qui est le mien. S'il avait simplement lu la seconde partie de Textualité et intertextualité des contes consacrée à une analyse textuelle des contes de Perrault (Heidmann \& Adam 2010), DA s'en serait peut-être rendu compte.

Mais, pour lui, mon œuvre de chercheur est caractérisée par «l'absence de cadres théoriques et épistémologiques homogènes» : "l'empan conceptuel développé par Adam sur la base de toutes ces disciplines pour l'analyse du texte est trop large pour fonder un ensemble structuré et articulé de concepts invariables, canoniquement fixés ». Je me demande si DA sait ce que c'est que la recherche. Lorsqu'il concède que "Certes l'idée d'une dynamique évolutive dans la théorisation est nécessaire pour parvenir jusqu'aux principes généraux constants et réguliers », DA ne le fait que pour ajouter aussitôt : «mais à condition que l'échafaudage de la théorie reste intact» et pour dénier à mes travaux le moindre échafaudage théorique.

Pourtant, ma théorie des modules ou plans d'analyse, sur laquelle je fonde ma conception de la complexité des textes, n'a pas considérablement varié depuis le milieu des années 1980. J'ai affiné la description des différents modules et leurs articulations, mais « l'échafaudage de la théorie » était déjà là et les concepts descriptifs de base également (progression thématique, anaphores, organisateurs textuels et connecteurs, temps verbaux et isotopies, chaînes d'actes de discours, théorie des séquences, etc.). Si DA ne veut pas le voir, je n'y peux strictement rien et je ne cherche pas ici à le convaincre. Ce sont les lecteurs de son article que je cherche à convaincre de la cohérence scientifique, bien sûr dynamique et évolutive, de mes travaux. C'est pour cela que j'ai toujours tenu à préciser, dans les diverses éditions successives de mes livres, les points d'évolution de ma théorie et mes choix terminologiques.

\subsection{Reconstructions imaginaires et dérives polémiques}

Le comble est atteint quand DA se permet d'effectuer une reconstruction aberrante de mes travaux :

Autre aspect du problème, que nous ne ferons également que signaler : les variations de l'attitude d'Adam à l'égard des catégories descriptives qui jouent sur un binarisme redondant : chaque concept-fétiche a son cadre théorique. La catégorie des «types de textes » est solidaire de la grammaire de texte, celle de la séquentialité de la pragmatique textuelle, celle des genres de la linguistique textuelle, et pour terminer celle de la généricité de l'analyse linguistique du discours. Il n'y a donc pas que les orientations théoriques et épistémologiques qui changent, mais les catégories aussi. [Sic pour la ponctuation.]

Certes la «solidarité » entre typologies de textes et grammaire de texte peut être invoquée historiquement (je l'ai expliqué à de nombreuses reprises) car l'une et l'autre se développent au milieu des années 1970. Mais la question des typologies 
de texte est précisément ce que j'attaque en élaborant ma théorie des séquences qui n'a de sens que dans le cadre plus large de la linguistique textuelle dont mon livre de 1990 dessine les grandes lignes. Je me suis expliqué longuement, dans l'édition 1999 de La linguistique textuelle, sur mon hésitation à parler de pragmatique textuelle et sur le fait que c'est un débat avec les développements de la pragmatique linguistique de Moeschler et Reboul qui m'a poussé à renoncer à cette appellation. Les séquences ne sont donc pas plus « solidaires » avec la pragmatique textuelle qu' avec la linguistique textuelle ou l'analyse textuelle des discours. La question des genres n'est pas, chez moi, couplée par fétichisme binariste avec la " linguistique textuelle ", ni la généricité avec "l'analyse linguistique du discours » (DA voulait probablement écrire l'analyse textuelle des discours). Pour moi, qui suis sur ce point très proche de Rastier, comme je l'ai clairement répété de nombreuses fois, le concept de genres fait le pont entre la linguistique textuelle et l'analyse de discours. C'est donc un concept du discours et pas de la linguistique textuelle. Je n'ai donc jamais pu écrire et penser ce que DA me fait dire ! Cette construction fallacieuse lui permet ensuite de réduire mon travail à un « binarisme redondant $»$.

Un dernier exemple. Après avoir cité des lignes d'un texte de moi non référencié en bibliographie (1991?) :

Adam propose de substituer aux typologies textuelles, une typologie basée sur la séquentialité. Son point nodal : «Les textes sont des structures tellement diverses et complexes qu'il est impossible d'en établir une typologie sauf par commodités pédagogiques illusoires. En revanche, on peut repérer des segments de plus petite taille, généralement composés de plusieurs phrases : les séquences. »(1991:9?).

DA formule ce commentaire époustouflant :

Deux choses sont à signaler dans cette citation. La première : les régularités des textes n'existent pas, la deuxième, conséquence directe de la précédente : la séquence est l'unité constituante du texte, seule capable de rendre compte de son hétérogénéité.

En quoi, cet extrait qui concerne uniquement la question des niveaux de possibilité de typologisation (niveau trop élevé à mon sens dans les « typologies de textes » et pertinent seulement au niveau séquentiel) veut-il dire que « les régularités des textes n'existent pas »? C'est absurde, car précisément la linguistique textuelle ne se justifie que parce que des régularités de rangs divers existent (phénomènes d'anaphore et de coréférence, de progressions thématiques, de connexion, de portée, structures périodiques de phrases, séquences textuelles et plans de textes, plans d'énonciation, etc.). Je ne dis rien de plus dans cette citation que le fait que les typologies globales de textes sont sinon impossibles, du moins réductrices. C'est pour cela que j' affirme, dans diverses mises au point, que les seules macro-régularités observables et typologisables sont les genres discursifs !

Je ne peux pas continuer à reprendre mot à mot des propos aussi approximatifs. Je signale seulement que le titre de la cinquième proposition donné par DA « La catégorisation générique se fait par airs de famille, au sein d'un système de genres » est celui de nos articles de 2004 et 2007, mais pas celui de la version 2009 de nos thèses : « Les genres n'existent qu'au sein d'un système de genres ». Or cette modification a un sens. Travailler, c'est corriger dans le détail ce qu'on a écrit, dans un souci de précision. Cette précision n'est jamais donnée, elle n'est conquise qu'au prix d'un travail continuel. S'il avait fait attention à ce type de correction, DA aurait été 
amené à modérer son emballement sur la question des « airs de famille » wittgensteiniens. Je renvoie les lecteurs sérieux à la lecture du bas de la page $18 \mathrm{du}$ Texte littéraire. Ils constateront que la question posée est celle de l'identification non pas numériquement computationnelle, mais humainement plus floue des genres :

L'identification d'un genre n'est pas un raisonnement abstrait, fondé sur le repérage d'ensembles de propriétés définies. Il s'agit plutôt de regroupements par « airs de famille ». Les genres sont des catégories définissables par des tendances ou des gradients de typicalité, par des faisceaux de régularités et des phénomènes de dominante. (Adam \& Heidmann 2009: 18)

Une dernière citation résumera à elle seule la polémicité du texte de DA. Il n'hésite pas à conclure ses trois premières parties par ces mots terribles, en dépit des modalisations qui n'atténuent en rien la violence de la charge :

Il n'y a donc pas que les orientations théoriques et épistémologiques qui changent, mais les catégories aussi. C'est ce qui pourrait peut-être expliquer pourquoi les réflexions d'Adam n'ont jamais pu s'ériger en théorie qui cherche à rassembler, ordonner et unir ses concepts, et les organiser autour d'un petit nombre d'idées-forces comme la marque d'une heuristique qui engage une réflexion sur les rapports qui unissent les catégories descriptives à un cadre théorique solide et inflexible.

On pourrait penser que c'est assez, mais DA ajoute une couche savoureuse :

Ce manque d'autonomie épistémologique, qui oblige à revenir sans cesse sur la plausibilité des catégories, des hypothèses et des cheminements qui ont conduit à l'élaboration des schémas et des modèles, contrarie la cohérence de son projet scientifique, et pourrait marquer ses limites, ou restreindre sa portée.

\section{Pour conclure}

S'il me restait un peu d'humour et de bonne humeur, je pourrais remercier DA et la revue Pratiques, d'avoir, en cette période de début de retraite marquée par plusieurs hommages, pris soin de m'éviter d'avoir la grosse tête. Se faire ramasser ainsi, à mon âge, dans une revue à laquelle j'ai collaboré pendant presque 40 ans, ne peut qu'être bénéfique pour mon équilibre mental et me rendre plus offensif et exigeant. C'est ainsi, je l'espère, que les lecteurs comprendront le ton de ma réponse à quelqu'un qui ne respecte pas, par manque de travail et d'ouverture intellectuelle, les principes du dialogue académique. Je n'ai pas cherché ici à entrer en débat avec lui ; j'ai seulement voulu attirer l'attention sur une stratégie discursive que je trouve déplorable.

Je laisse Érasme conclure, sans espoir que DA puisse jamais comprendre cette maxime : "De même que, tant que nous vivons, nous ne cessons de travailler à nous rendre meilleurs, de même nous ne cessons de corriger et de compléter les cuvres de notre esprit qu'en cessant ainsi de vivre ». 


\section{Bibliographie}

ACHARD-BAYLE, G. (2013) : «Texte et discours se comprennent-ils ? », in Genres et textes. Déterminations, évolutions, confrontations, M. Monte \& G. Philippe éds., Presses universitaires de Lyon, à paraître.

ADAM J.-M. (2013) : «Macro-propositions, séquences et plans de textes : discussion des propositions d'André Avias », Semen 34, à paraître.

— (2012) : «La brève comme récit minimal : les Nouvelles en trois lignes de F. Fénéon ", in Le récit minimal, S. Bedrane, F. Revaz, M. Viegnes (éds.), Paris, Presses de la Sorbonne Nouvelle, 33-44.

- (2011a): Genres de récits. Narrativité et généricité des textes, Louvain-laNeuve, Academia-L'Harmattan.

- (2011b [1992]) : Les textes : types et prototypes, Paris, A. Colin.

- (2011c [2005]) : La linguistique textuelle, Paris, A. Colin.

— (2010) : «De la duplicité des modalités de communication d'un parti nationaliste suisse : l'UDC/SVP », avec la collaboration de Stéphanie Pahud, in: Le Discours du nationalisme en Europe, G. Komur-Thilloy et A. Celle (éds.), Paris, Ed. L'Improviste, 287-300.

- (2008a) : «La construction textuelle de l'explication : marqueurs et portées, périodes et séquences ", in L'explication. Enjeux cognitifs et interactionnels, C. Hudelot, A. Salazar Orvig \& E. Veneziano éds., Peeters, Louvain, 23-40.

— (2008b) : «Énonciation et narration. Fragments de rhétorique chiraquienne», in Argumentation et narration, E. Danblon, E de Jonge, E. Kissina \& L. Nicolas éds., Éditions de l'Université de Bruxelles, 19-28.

- $(2008 \mathrm{c})$ : « Les lettres asilaires de Gaspard Corpataux, poèmes de l'enfermement », avec la collaboration de Vincent Capt, in Art Brut fribourgeois, Catalogue de l'exposition, Collection de l'Art Brut \& éd. La Sarine, Lausanne-Fribourg, 72-79.

- (2006) : «Effacement énonciatif et diffraction co-textuelle de la prise en charge des énoncés dans les hyperstructures journalistiques », avec la collaboration de Gilles Lugrin, Semen 22, 127-144.

- (2005) : "Variété des usages de SI dans l'argumentation publicitaire », in Argumentation et communication dans les médias, Marcel Burger \& Guylaine Martel éds., Nota Bene, Québec, 81-109.

— (2004a) : "Quand dire "Vive le Québec libre !" c'est faire l'Histoire avec des mots ", in Discours et constructions identitaires, D. Deshayes \& D. Vincent éds., Presses de l'Université Laval, Québec, 13-38.

— (2004b) : «Une approche textuelle de l'argumentation : "schéma", séquence et phrase périodique ", in L'argumentation aujourd'hui, M. Doury \& S. Moirand éds., Presses de la Sorbonne Nouvelle, Paris, 77-102.

- (2003a) : «Discours de combat et argumentation épidictique. De Gaulle, discours du 6 juin 1944 », avec la collaboration de Th. Herman, Champs du signe 15, Toulouse, Éditions Universitaires du Sud, 137-157.

- (2003b) : " Note de lecture d'Arts et sciences du texte de François Rastier », Revue romane 38-2, Copenhague, 322-332.

- (2001a) : « Types de textes ou genres de discours? Comment classer les textes qui disent de et comment faire ? », Langages 141, 10-27.

- (2001b) : « Entre conseil et consigne : les genres de l'incitation à l'action », Pratiques 111-112, 7-37.

— (2001c) : «La presse écrite : typologies, genres et mélanges de genres », Études de Lettres $n^{\circ} 3-4,5-11$. 
— (2000a) : «L'hyperstructure : un mode privilégié de présentation des événements scientifiques? », Cahiers du Cediscor $6:$ "Rencontres discursives entre sciences et politique dans les médias », avec la collaboration de Gilles Lugrin, Paris, Presses de la Sorbonne Nouvelle, 133-149.

— (2000b) : «La presse écrite : typologies, genres et mélanges de genres », Études de Lettres 3-4, 5-11.

— (2000c) : «Genres de la écrite et analyse de discours », Semen 13, 7-14.

- (2000d) : "Reformulation, répétition et style périodique dans l'appel du 18 juin », avec la collaboration de Thierry Herman, Semen 12, 11-30.

- (1999) : La linguistique textuelle. Des genres de discours aux textes, Paris, Nathan, coll. FAC.

- (1997a) : " Approche micro-linguistique d'une catastrophe naturelle », Sciences de la Société 41, Presses Universitaires du Mirail, Toulouse, 125-144.

- (1997b) : «Unités rédactionnelles et genres discursifs : cadre général pour une approche de la presse écrite », Pratiques 94, 3-18.

- (1990) : Éléments de linguistique textuelle, Bruxelles, Mardaga.

ADAM, J.-M. \& BONHOMME, M. (2012 [1997]) : L'argumentation publicitaire, Paris, Armand Colin.

— (éds.) (2000) : Analyses du discours publicitaire, Toulouse, Éditions Universitaires du Sud, coll. Champs du Signe.

ADAM, J.-M. \& GOLDENSTEIN, J.-P. (1976) : Linguistique et discours littéraire, Paris, Larousse, coll. L.

AdAM, J.-M. \& HeIDMAnN, U. (2009) : Le texte littéraire. Pour une approche interdisciplinaire, Louvain-la-Neuve, Academia Bruylant.

— (2006) : "Six propositions pour l'étude de la généricité », avec la collaboration d'Ute Heidmann, in Le Savoir des genres, R. Baroni \& M. Macé éds., La Licorne $\mathrm{n}^{\circ} 79$, Presses Universitaires de Rennes, 21-34.

- (2004) : «Des genres à la généricité », avec la collaboration d'Ute Heidmann, Langages 153, Paris, Larousse, 62-72.

ADAM, J.-M. \& VIPREY, J.-M. (éds.) (2009) : « Corpus de textes. Textes en corpus », Corpus 8, Nice, Presses de 1'Université de Nice-Sophia Antipolis.

AviAS, A. (2013) : « La macro-propositions : unité compositionnelle variable », Semen 33.

Charaudeau, P. (2013) : « Le dispositif comme fondateur d'un genre : la Controverse », in Genres et textes. Déterminations, évolutions, confrontations, M. Monte \& G. Philippe éds., Presses universitaires de Lyon, à paraître.

Condillac, Etienne Bonnot de (2002 [1803]) : L'Art d'écrire, Orléans, Editions Le Pli.

Heidmann, U. \& AdAm, J.-M. (2010) : Textualité et intertextualité des contes. Perrault, Apulée, La Fontaine, L'héritier, Paris, Classiques Garnier.

JAKobson, R. (1980) : Dialogue, avec Krystyna Pomorska, Paris, Flammarion.

MONTE, M. (2013) : «Les textes entre variation et régularités », in Genres et textes. Déterminations, évolutions, confrontations, M. Monte \& G. Philippe éds., Presses universitaires de Lyon, à paraître.

SAINT-GERAND J.-Ph. (2012) : Compte-rendu de lecture de Genres de récits, in Questions de communication 22, accessible en ligne sur : http://questionsdecommunication.revues.org/6988.

PAVEAU, M.-A. \& et S ARFATI, G. E. (2003) : Les grandes théories de la linguistique, Paris, A. Colin.

VIPREY, J.-M. (2011) : «Compte-rendu de lecture de La linguistique textuelle 2011 », Semen 32, 182-185. 